\title{
Information et Communication: Dix Chantiers Scientifiques, Culturels et Politiques
}

\author{
Dominique Wolton \\ Laboratoire communication et politique, CNRS, Paris
}

\begin{abstract}
Resumo: O texto propõe, através de dez pontos selecionados, um olhar sobre questões da comunicação e da informação, sendo consideradas questões antropológicas, culturais e políticas, que podem diferenciar os conceitos apresentados. Uma dissociação entre informação e comunicação é percebida e analisada em contexto de identidades culturais coletivas.
\end{abstract}

Palavras-Chaves: Comunicação, Informação, Identidades, Cultura, Política

Qu'apportent, de mon point de vue de chercheur, les sciences de l'information et de la communication depuis un demi-siècle ? Cinq acquis, apparemment modestes, mais qui sont autant d'outils pour penser une des questions anthropologiques les plus compliquées : qu'est-ce communiquer?

\section{La dissociation entre information et communication}

Les deux ne sont pas synonymes, même si depuis cinquante ans les progrès techniques concernent la capacité à produire et distribuer de plus en plus vite un nombre considérable d'informations de toute nature. Chacun sait bien que la difficulté essentielle est dans la communication, qui implique la relation, c'est-à-dire la prise en compte de l'Autre. Avec l'information, on reste du côté du signe, de l'unité cognitive et symbolique. Avec la communication, on butte sur l'Autre, le récepteur. Celui qui n'entend pas, ne comprend pas, refuse, recule, recode, s'éloigne, transforme. L'épreuve reste l'Autre, quelle que soit la nature, la forme de l'information, l'identité et l'échelle du destinataire. La croissance de l'information et la multiplication, comme 
l'hétérogénéité des récepteurs rendent finalement visible cette dissociation entre information et communication. Celle-ci a toujours existé, mais pendant des siècles la rareté de l'information, et la difficulté de sa transmission, autant que l'échelle relativement limitée des échanges entre les hommes et les sociétés étaient telles que l'on croyait de bonne foi que l’information créait de la communication. C'est paradoxalement l'explosion de l'information, les progrès technologiques, ainsi que les échelles de réception qui rendent plus visible cette dissociation.

\section{Transmettre n'est pas communiquer}

Un plus grand nombre d'information, transmis de manière très efficace et rapide, par un plus grand nombre de systèmes techniques n'assure pas forcément une meilleure communication. Preuve qu'il ne suffit plus de disposer d'un grand nombre d'informations et beaucoup de tuyaux pour créer de la communication. L'essentiel n'est pas la transmission, mais la communication c'est-à-dire le partage d'un minimum de valeurs pour accepter de débattre et de partager ce qui est reçu. C'est pour prendre en compte de ce phénomène inattendu d'une distorsion entre le nombre d'informations diffusées et les décalages dans la réception que dans mon dernier livre, « l'Autre mondialisation », je parle de trois mondialisations. La première, politique, est liée à la construction du cadre démocratique de l'Onu à la sortie de la guerre. La seconde est économique. La troisième, demain, concerne l'émergence du couple culture-communication. Les deux sont devenues indissociables, faisant de ce couple, comme on le voit en accéléré depuis la fin de l'affrontement Est-Ouest, un des facteurs de paix et de guerre, les plus importants. L'enjeu de cette troisième mondialisation est la capacité à organiser démocratiquement, la cohabitation culturelle, à l'échelle des sociétés et du monde. C'est-à-dire de réfléchir aux conditions pour établir un minimum de communication en dépit de la diversité des cultures des récepteurs. Tout faire pour éviter que la facilité des échanges et la visibilité 
plus grande du monde, au lieu de rapprocher les points de vue, soit au contraire l'occasion d'une insupportabilité plus grande. Les différences culturelles, sociales et politiques deviennent le ferment de nouveaux conflits. Car tel est le paradoxe : il n'y a jamais eu autant d'échanges et autant d'incompréhension. Tout simplement parce que la fin des distances culturelles révèle l'étendue des distances culturelles. Plus que jamais la révolution de l'information et de la communication, où se mêlent progrès techniques, enjeux économiques, utopies sociales fait apparaître la différence entre deux grandes philosophies de la communication. Une technologique et économique, et l'autre humaniste et démocratique. La différence reste essentielle entre ceux qui, finalement, croient que les hommes au bout des techniques et des échanges, se comprennent, et ceux qui en doutent. Et ceux-là, remettent la politique, au sens de la définition de règles nécessaires d'arbitrage entre des visions différentes du monde au cœur des rapports entre information, communication et société.

$\mathrm{Si}$ dans les valeurs occidentales, et même ailleurs, l'information, la communication restent des références humanistes et démocratiques cela ne suffit pas à simplifier ou améliorer les rapports humains et sociaux. L'opposition entre ces deux philosophies de la communication, humaniste et technique, renvoie aux deux conceptions normatives et fonctionnelles de la communication.

\section{Communiquer : le temps et l'expérience}

$\mathrm{Au}$ bout des images des réseaux, des systèmes, les hommes doivent passer et perdre du temps, pour se voir, se rencontrer afin que quelque chose, finalement, se passe et se décide. C'est vrai aussi bien dans la vie scientifique que dans celle des affaires et de la politique. D'ailleurs depuis un siècle les déplacements physiques des hommes ont cru proportionnellement à la performance de toutes les techniques de communication qui devaient justement permettre de les réduire. L'épreuve de la rencontre avec autrui, 
passe par la rencontre et le temps. Finalement après tous les miracles techniques, les prolongements de nos sens, de nos capacités cognitives nous sommes avant tout des êtres sociaux. L'expérience reste le fondement des rapports humains. La technique ne comble ni le doute métaphysique ni la rencontre physique requise pour toute situation de communication.

\section{L'apport des sciences de l'information et de la communication}

Certes elles sont liées à la compréhension de la place actuelle de l'information et de la communication dans des sociétés ouvertes où l'on constate un entremêlement permanent entre l'idéal humaniste et démocratique et les intérêts du marché, entre les techniques et la politique. Mais elles ont une autre utilité. Elles déplacent les frontières et les problématiques des autres disciplines traditionnelles qui de la philosophie à la sociologie, de l'anthropologie à la linguistique, de l'histoire à la science politique, de la géographie au droit et à l'économie.... ont depuis longtemps pensé, dans leur vocabulaire, l'information et la communication. Les sciences de l'information et de la communication obligent ces disciplines à réexaminer leur approche des rapports entre information, culture, communication, connaissance et société. Les disciplines plus anciennes ne sont pas « dépassées » par ce nouveau domaine de connaissance scientifique : il n’y a pas d'échelle « du progrès » entre les «vieilles » et les « nouvelles » sciences. Simplement le regard qu'elles apportent sur une vieille question, celle de l'information et de la communication, à l'aune des changements technologiques économiques, culturels et sociaux survenus depuis près d'un siècle éclaire autrement la problématique traditionnelle. Par comparaison, la psychanalyse n'a pas invalidé la psychiatrie, la médecine, la linguistique et l'ensemble des sciences qui s'occupent du langage et du rapport au monde et à autrui. Elle a obligé à prendre en compte cette nouvelle approche pour réexaminer raisonnements et problématiques. Il en est de même pour les sciences de l'information et de la communication. Elles invitent à penser 
autrement les objets de connaissance et les représentations. À la place qui est la sienne, cette discipline n'a aucune prétention à se substituer aux autres. Elle contribue simplement à penser différemment les réalités sociales, politiques, culturelles, psychologiques.

\section{Le surgissement des identités culturelles collectives}

Les sciences de la communication permettent de voir plus rapidement cet enjeu, l'un des plus importants du XXIe siècle. Non seulement le monde, devenu plus petit n'est pas forcément plus compréhensible, mais surtout en rendant plus visible les différences culturelles, au sens des langues, religions, systèmes symboliques, représentations... il oblige à un gigantesque effort de tolérance et d'intercompréhension qui rejoint d'ailleurs les idéaux de la première mondialisation dans la charte de l'Onu de 1948 : la nécessité d'une communauté internationale respectueuse des différences culturelles et religieuses. Simplement le contexte a changé en un demi-siècle. Dans un monde plus visible, où les échanges ne cessent de croître les identités culturelles collectives s'affirment de plus en plus et deviennent un facteur d'antagonisme. La première mondialisation posait le principe du respect des cultures. La troisième l'impose, sous peine de faire de la culture un des agents conflictuels, les plus actifs du siècle à venir. Autrement dit, si le village global physique n'a pas apporté la compréhension et la communication mondiale, il a mis au contraire au cœur des rapports humains et sociaux, la problématique et l'identité culturelle collective. Les identités culturelles collectives ont toujours existé, mais elles n’ont jamais été aussi visibles, et revendiquées à la fois par l'idéal démocratique mondial, et par les sociét és qui menacées par l'ouverture assurées par la mondialisation les revendiquent de plus en plus. Il suffit de voir ce qu'il s'est passé en Iran depuis 1979 et dans de nombreux autres pays. Pendant plus d'un siècle les inégalités économiques et sociales ont été au cœur de conflits. Aujourd'hui c'est aussi le cas avec la culture, entendue comme l'ensemble des éléments du patrimoine et de la réalité contemporaine qui 
permettent de comprendre le monde, de s'en faire une représentation, et d'agir. Avec la mondialisation on assiste simultanément à une sorte de lutte de classes à l'échelle du monde et au surgissement des risques d'une lutte des cultures. La question de la cohabitation culturelle c'est-à-dire la gestion pacifique des identités culturelles collectives au plan mondial est un nouveau défi. Jamais la question de l'Autre, comme horizon de la communication, ne s'est posée à cette échelle, ni à cette vitesse. En réalité nous avons à la fois des systèmes techniques extrêmement performants, et une obligation moins facile à satisfaire : penser la place croissante des identités culturelles collectives dans un monde ouvert où la facilité, et la vitesse des échanges n'est pas synonyme de plus de tolérance. C'est en cela que les sciences de l'information et de la communication touchent, comme la plupart des sciences humaines et sociales à la question essentielle de la paix et de la guerre.

\section{Dix chantiers}

Dans le foisonnement des enjeux scientifiques culturels sociaux et politiques qui traversent le champ de la communication, dix chantiers permettent de valoriser le rôle de la connaissance.

1. Ne jamais rester au niveau des pratiques et des discours mais essayer d'avoir une approche théorique pour hiérarchiser, mettre en perspective et relativiser.

Par exemple, bien distinguer les deux philosophies de la communication sous-jacentes à de nombreux projets et promesses. Celle qui insiste sur la technique et finalement le " progrès » économiques. Celle qui est d'abord sensible à une perspective humaniste et finalement en filigrane, aux enjeux politiques. Dans un cas, on parle plutôt de progrès, d'adaptation, de révolution technique. De l'autre on réfléchit plutôt dans une perspective anthropologique et critique nécessairement dubitative. Les deux démarches sont utiles et finalement complémentaires, à condition de ne pas les hiérarchiser. Il est évident que le progrès technique et les enjeux économiques poussent 
davantage vers le thème de la société de l'information, alors qu'une approche plus critique et sociétale est davantage sensible aux inégalités et aux interrogations sur les limites des modèles cognitifs et rationnels liés à l'expansion des réseaux et des théories de la société de l'information. Distinguer une approche humaniste et technique de la communication retrouve l'opposition entre deux philosophies de la communication caractérisées dans Penser la communication : la philosophie normative et la philosophie fonctionnelle de la communication.

2. Associer les thèmes liés aux techniques et usages aux questions plus théoriques des rapports entre connaissance et représentation, information et opinion, stéréotypes et idéologies. Les échanges plus nombreux ont-ils un impact sur ces « infrastructures » culturelles et mentales qui structurent en bonne partie notre rapport au monde ? D'autant que les individus, comme les sociétés, maintiennent des dispositifs cognitifs et culturels très complexes entre eux et le monde. Dispositifs qui filtrent et recodent tout ce qui vient de l'extérieur. Pourquoi, par exemple, la multiplication des informations qui rend le monde plus visible, en tout cas dans les pays démocratiques ne suffit-elle pas à le rendre plus compréhensible, moins violent ? Hier on pensait que l'absence d'information expliquait en partie la violence du monde. Aujourd'hui on constate que l'abondance d'information ne change rien à sa violence. De même que l'horizon de la communication reste l'incommunication, de même faut-il mettre en perspectives les triomphes de l'information et de la communication avec les infrastructures cognitives, imaginaires et symboliques qui structurent le rapport au monde. Passer de la rationalité des échanges, à la complexité de représentations des effets et des idéologies. Pourquoi, les rumeurs augmentent-elles aussi vite que les informations et les connaissances, chargées au contraire de les relativiser ? Pourquoi les stéréotypes et les idéologies sont-elles imperméables à toute rationalité communicationnelle? 
3. Valoriser la problématique du récepteur. L'augmentation des volumes d’information échangés ne crée pas forcément une meilleure communication, tout simplement, parce que émetteur et récepteur ne sont jamais en ligne. Les récepteurs sont de plus en plus autonomes par rapport aux messages, au fur et à mesure que ceux-ci sont plus nombreux et hétérogènes. Qu'est ce qui résiste et se déforme dans les processus de communication interpersonnels ou collectifs ? Internet qui dans un premier temps, par exemple a été perçu comme un accélérateur de communication se révèle être un fantastique moulin à « rumeurs ». Certains y font intentionnellement circuler des informations fausses, et mais surtout l'imaginaire se saisit de ces flux ininterrompus d'information, et demain d'images, qui auto-génèrent des intentionnalités inattendues. L'homme n'était à peu près rationnel à l'égard de l'information, que lorsqu'elle était rare. La complexité des rapports entre information, culture, représentation, idéologie réapparaît dès lors que les informations sont nombreuses. Le volume d’information et la liberté d'accès ne créent pas forcément plus de rationalité dans le comportement du récepteur.

4. L'individualisation et l'interactivité devaient permettre l'émergence d'une société plus personnalisée : on retrouve au contraire, les problèmes collectifs. L’individualisation des techniques, des messages, des services, des marchés, n’arrive pas à venir à bout de la société et de son épaisseur. Autrement dit, plus les relations s'individualisent plus le poids de la société, des valeurs, des idéologies, des intérêts collectifs, du passé... refait surface. À la rationalité souhaitée des relations individuelles s'oppose finalement assez vite le poids de la société avec son épaisseur historique, culturelle et religieuse. On pensait les individus désireux de s'en affranchir, on voit qu'ils ne peuvent y échapper... On croyait aller vers davantage d’individualisation, on retourne vers le collectif. Ce sont les contradictions de ce que j’appelle la société individualiste de masse. Et cette contradiction rencontrée au niveau des États Nations se retrouve encore plus dans le cadre de la mondialisation où chacun 
veut à la fois appartenir à la modernité et à la mondialisation des échanges, sans abandonner ses identités culturelles collectives, ses histoires, son patrimoine... Le surgissement de la culture au sens large comme enjeu des conflits depuis une génération illustre, les limites du processus de modernisation et de rationalisation qui devait se produire avec l'émergence du « village global ». Pour une part, les individus et les peuples veulent bien être inscrits dans la modernité communicationnelle, mais d'autre part ils restent viscéralement attachés à leurs racines culturelles et symboliques qui sont parfois complètement antithétique des premières.

5. Réfléchir au statut de l'Autre rendu plus visible par la généralisation des échanges. Non seulement la communication ne rapproche pas forcément des points de vues, mais elle peut même amplifier l'incommunication. Comment gérer l'altérité dans un univers où la communication a toute sa place, mais sur un mode où domine finalement la rationalisation ? Valoriser la problématique de l'Autre dans la communication, c'est aussi revenir sur les glissements de sens du mot information. Celui-ci fut d'abord lié à une revendication politique : la liberté d'information comme condition de la démocratie et le complément de la liberté de conscience. Elle fut ensuite le symbole de la presse : le droit de savoir ce qu'il se passe. L'information ici est liée à l'événement c'est-à-dire à ce qui surgit et fait rupture. Et il y a maintenant un troisième sens du mot information, apparemment en continuité avec les deux premiers, mais en réalité complètement distant et lié aux systèmes d'information. Il est beaucoup plus proche de l'économie et de la gestion du monde complexe : c'est l'information fonctionnelle. L'information des systèmes d'information n'est pas synonyme de l'information politique et de l'information événement. C'est même parfois l'inverse, car elle s'appuie sur une certaine continuité et rationalité. Or la problématique de l'Autre dans la communication, renvoie beaucoup plus aux deux premiers sens de l'information qu'au troisième sens. Autrement dit les difficultés de la communication au sens de la place que l'on accorde à l'Autre obligent à 
réexaminer les différents sens du mot information notamment dans les activités d'éducation, de recherche, d'échanges interculturels...

6. Socialiser les questions d'information et de communication. Non pas simplement pour rappeler l'importance des conditions sociales et culturelles inhérentes à toutes situations de communication mais pour rappeler qu'il n'y a pas de communication sans histoire. Au bout des réseaux existent des hommes, des cultures et des sociétés qui rapidement feront surgir les multiples formes d'altérité. Débattre des enjeux, économiques et politiques des systèmes d'information, mais également socio-culturels, c'est réduire les risques d'une explosion ultérieure. Réguler, politiser les questions d'information et de communication, ce n'est pas reculer, ou régresser, ou perdre du temps, c'est éviter que soient rejetés un jour les industries et les intérêts, mais aussi les valeurs de liberté et d'émancipation qui sont inhérentes à l'information et la communication. Ouvrir, socialiser, débattre pour éviter qu'un jour les luttes altermondialistes, par exemple, dans leur travail critique n'identifient ces industries à une simple logique capitaliste et dominatrice. Éviter ainsi que tout ne soit réduit à une problématique simple, et rassurante, mais seulement en partie vraie, "d'impérialisme culturel ». Réguler, ouvrir des débats, évaluer les expérimentations, comparer : tout faire pour sortir de la technique, des usages et de l'économie et permettre à la société, aux imaginaires de se saisir à leur tour de cette « révolution ». Celle-ci doit se socialiser. Elle ne peut s'imposer en restant sur le seul versant de la performance et de la rationalité. C'est en ne perdant jamais de vue le lien fort existant entre information, communication et imaginaire, utopies, idéologies et représentations que l'on pourra progressivement introduire les systèmes d'information dans la communauté internationale et éviter les risques violents de rejets dont ils peuvent être l'objet. Socialiser les questions d'information et de communication, ce n'est pas perdre du temps, c'est en gagner.

7. Puisque dans la communication l'essentiel n'est pas du côté des techniques et de l'économie, mais du côté des modèles culturels, il est 
essentiel d'introduire une perspective historique et comparative. Historique pour voir des changements d'échelle et de comportement, ainsi que les discontinuités et ruptures dans les discours tenus soit sur les techniques, soit sur les modèles sociaux et culturels de la communication. Rien de pire que de croire que l'histoire commence avec les modèles et les politiques de communication contemporaines. Quant au comparatisme, il permet d'introduire concrètement à la diversité culturelle, grand enjeu du XXIe siècle. Si l'on ne veut pas que le modèle occidental de communication, qui s'est répandu dans le monde, en mélangeant une certaine prétention à l'universel, son propre modèle et ses intérêts techniques et économiques, ne soit rejeté au titre de l'impérialisme occidental, il faut au plus vite s'ouvrir aux autres modèles culturels et politiques de communication. Non pas pour créer une sorte de cosmopolitisme artificiel mais pour montrer que la prétention à l'universalisme de l'occident n'est pas un simple occidentalisme. Et pour bien suggérer que dans une perspective normative, et non fonctionnelle de la communication, la plus grande transparence du monde suppose aussi la cohabitation culturelle au niveau mondial. Privilégier une approche normative, et non fonctionnelle de la communication, c'est privilégier la dimension culturelle par rapport aux deux autres, techniques et économiques. C'est aussi admettre le relativisme de notre modèle culturel occidental de la communication. C'est enfin le moyen de valoriser la problématique de la cohabitation culturelle. En un mot donner un peu de profondeur et de perspective, à un champ d'activité, et de représentations, trop réduit aux mutations contemporaines.

8. Au niveau des principes la distinction entre les dimensions normatives et fonctionnelles de la communication, perspective humaniste et technique sont d'autant plus nécessaires à maintenir que dans la réalité, tout est beaucoup plus mêlé. C'est même ce mélange entre fonctionnalités et valeurs qui à la fois plaît à chacun d'entre nous et fait croire à certains qu'il n'y a rien à penser avec la communication puisque «ça marche ». Mais le fait que les 
marchés se développent et que les individus « en redemandent » ne suffit pas, car la subjectivité et l'imaginaire ne sont jamais loin dans les rationalités. Par exemple, dans la formation aux métiers, ne pas choisir entre la dimension théorique et pratique, mais apprendre les deux. Le problème n'est pas l'ambiguité de toute problématique de communication, mais le fait de nier cette dualité. Celle-ci doit rester visible pour que chacun sache à quoi s'en tenir. Rien de pire que de croire qu'entre la définition humaniste et technique, normative et fonctionnelle de la communication, une des deux dimensions doit finalement l'emporter. Les deux, existent toujours. Ne pas séparer les préoccupations normatives des réalités concrètes est le moyen de rappeler que tout ce qui touche à la communication ne peut se réduire à la performance technique ou la rationalité économique. Et réciproquement. Parler, écrire, filmer, échanger est toujours ambiguë et polysémique. Tant mieux.

9. Aujourd'hui, culture et communication sont inséparables. La culture n'existe pas sans une politique de diffusion à l'échelle plus ou moins mondiale et sans une attention à la réception. Et réciproquement, toute pratique de communication est majoritairement liée à la culture dominante de la modernité. Le lien entre les deux évite de séparer la « bonne culture » de la « mauvaise culture ». Avec le monde ouvert, la fin de l'affrontement Est-ouest, l'essor des techniques et des industries culturelles mondiales, culture et communication avancent de pair. Elles offrent à la fois, l'ouverture au monde ; une clé de compréhension de la réalité contemporaine autant que la nécessité de ne pas bouder le passé. La modernité de la communication n'est rien sans les cultures de la tradition. Ne pas les séparer a deux autres avantages : rappeler qu'il y a une autre culture que celle de la communication. Avec la mondialisation de la communication, les revendications d'identités culturelles collectives seront croissantes. Autrement dit dans les rapports de plus en plus imbriqués entre culture et communication, il ne faut pas oublier l'ambivalence fondamentale de la communication, entre fonctionnalité et normativité. Il ne 
faut pas non plus oublier les deux dimensions de la culture, individuelle et collective, moderne et traditionnelle.

10. Pour affronter toutes ces ambivalences, dualités, séductions le rôle des connaissances gratuites, de la recherche et de la formation est indispensable. Plus les discours publics, politiques et économiques parlent de la " révolution de la communication ", plus il faut introduire la distance critique apportée par la connaissance, l'école et l'université. Distance indispensable pour résister à la tyrannie des marchés et des techniques. L'espace de la connaissance et la formation offrent une altérité, par rapport à l'empirisme de la modernité de la technique et de l'économie. La communication requiert cette altérité fournie par le monde de la culture et de la connaissance pour résister à l'efficacité croissante des marchés. Maintenir les distances, est le seul moyen d'échapper au piège de la rationalité cognitive, technique et économique, sociale dominante. La communication, peut être plus que toute autre activité sociale, requiert à la fois ; la cohabitation de valeurs normatives et fonctionnelles ; la double référence à la technique et à la politique ; l'articulation entre l'individuel et le collectif. L'ironie, le doute, l'humour, la distance sont les armes nécessaires pour rappeler la complexité et l'ambivalence de toute situation de communication. Après tout chacun sait bien que l'idéal de la communication est la mise en rapport, l'échange et la compréhension mais qu'au même moment, les individus ou les collectivités font tout pour que cette mise en rapport ne réussisse pas réellement. «Plus ça marche plus on s'esquive ». Chacun recherche la communication et en même temps se ménage une marge de manœuvre d'autant plus nécessaire que les outils sont de plus en plus parfaits et les discours de plus en plus centrés sur la communication. On pourrait en arriver à oublier qu'il n'y a pas de communication sans échec, sans dérapage, sans extériorité... Au moment où le monde devient un village fini et fermé, souligner l'incertitude qu'implique toute situation de communication est le moyen de rappeler combien la communication reste synonyme de liberté. 
